\title{
Recherche de la toxicité de diverses souches de Bacillus thuringiensis Berliner vis-à-vis de trois espèces de limaces
}

\author{
JC Kienlen 1, C Gertz 1, P Briard 2, G Hommay ${ }^{1 *}$, J Chaufaux ${ }^{3}$ \\ 1 Laboratoire de zoologie, Inra, F-68021 Colmar cedex; \\ 2 Inra, domaine de la Grande-Ferrade, BP 131, F-33140 Pont-de-la-Maye; \\ ${ }^{3}$ Station de recherches de lutte biologique, Inra, La Minière, F-78285 Guyancourt cedex, France
}

(Reçu le 9 mars 1995 ; accepté le 24 mai 1996)

\begin{abstract}
Résumé - La toxicité de plusieurs préparations à base de Bacillus thuringiensis a été montrée vis-à-vis de la limace grise Deroceras reticulatum (Müller) et de l'escargot aquatique Biomphalaria alexandrina (Ehrenberg) par de précédents auteurs, laissant envisager une méthode de lutte microbiologique contre les gastéropodes. C'est pourquoi la toxicité de certains de ces produits commerciaux et de diverses souches non formulées ayant des activités insecticides différentes a été éprouvée sur trois espèces de limaces : $D$ reticulatum, Arion distinctus Mabille et Limax valentianus Férussac et comparée à celle de produits molluscicides de référence. Parmi les produits testés, deux étaient à base de $\beta$-exotoxine (Bitoxibacillin 5 et le surnageant $\mathrm{H1}$ ). Cependant, aucune préparation à base de Bacillus ne s'est montrée toxique vis-à-vis des trois espèces de limaces. Seuls les produits molluscicides ont confirmé leur efficacité contre les limaces.
\end{abstract}

\section{Deroceras reticulatum / Arion distinctus / Limax valentianus / Bacillus thuringiensis / molluscicide}

Summary - Research on the toxicity of various Bacillus thuringiensis Berliner strains to three species of slugs. Toxicity of several Bacillus thuringiensis preparations had been shown against the grey field slug Deroceras reticulatum (Müller) and the aquatic snail Biomphalaria alexandrina (Ehrenberg) by previous authors, letting view a method of microbiological control of gastropods. Therefore, the toxicities of some of these products and of several unformulated strains, having different insecticide activities, were studied on three species of slugs: D reticulatum, Arion distinctus Mabille and Limax valentianus Férussac, and compared to molluscicide products of reference. Amongst the products tested, two contained the $\beta$-exotoxin (Bitoxibacillin 5 and the surnatant H1). However, no strain of Bacillus showed to be toxic for the three slug species. Only the molluscicide products confirmed their efficiency against the slugs.

\section{Deroceras reticulatum / Arion distinctus / Limax valentianus / Bacillus thuringiensis / molluscicide}

\section{INTRODUCTION}

La plupart des dégâts de limaces observés en France dans les cultures de plein champ sont dus à la limace grise Deroceras reticulatum (Müller) et à la limace horticole Arion hortensis s I
Férussac (Hommay et Lavanceau, 1986), qui est en fait un complexe de trois espèces jumelles: $A$ hortensis Férussac, $A$ distinctus Mabille et $A$ owenii Davies. Par ailleurs, dans les serres, d'importants dégâts sont parfois commis par Limax valentianus Férussac sur les fleurs d'orchi- 
dées et sur d'autres plantes ornementales (Waldén, 1960 ; Moens, 1980 ; South, 1992). Cette espèce, originaire de la péninsule ibérique, a été involontairement disséminée par l'homme dans le nord de l'Europe et sur les autres continents. La lutte chimique est la principale méthode de lutte contre les limaces, cependant les matières actives utilisées actuellement présentent l'inconvénient de ne pas être spécifiques. Elles sont toxiques vis-à-vis des vertébrés et des mortalités sont parfois observées parmi la faune domestique et sauvage, lorsque les produits sont mal dispersés. Elles sont également toxiques visà-vis de nombreux invertébrés (vers de terre, entomofaune auxiliaire), à l'exception du métaldéhyde qui agit uniquement sur les gastéropodes. Bacillus thuringiensis Berliner est une bactérie qui possède une activité entomopathogène. Selon les souches, $B$ thuringiensis présente une activité contre les larves de certains lépidoptères, coléoptères ou diptères. Le pouvoir pathogène et la spécificité d'action de $B$ thuringiensis sont essentiellement déterminés par des protéines insecticides, les $\delta$-endotoxines, qui sont synthétisées sous forme d'inclusions cristallines au moment de la sporulation. La spécificité d'action de $B$ thuringiensis (Lereclus et Sanchis, 1989 ) et son innocuité vis-à-vis des plantes et des autres animaux, en particulier de la faune utile (pollinisateurs, auxiliaires), font que plusieurs souches sont utilisées comme biopesticides. Certaines souches de $B$ thuringiensis produisent des $\beta$-exotoxines qui possèdent des propriétés mutagènes et tératogènes. De ce fait, les produits à base de ces souches sont rigoureusement interdits à la commercialisation dans le monde occidental (Burgerjon, 1972). De nouvelles souches de $B$ thuringiensis découvertes ces dernières années ont une activité sur des organismes nuisibles autres que les insectes, tels que les nématodes (Poinar et al, 1990) et les acariens (Royalty et al, 1990). Selon RajnchapelMessaï (1993), la société californienne Mycogen a ainsi développé des brevets contre de nouveaux insectes tels que la mouche domestique et les blattes, mais également des protozoaires, des nématodes, des trématodes et des acariens. Une action toxique des produits formulés à partir de spores et de cristaux de $B$ thuringiensis contenant de la $\delta$-endotoxine (Dendrobacillin, Thuricide HP, Dipel) ou de la $\beta$-exotoxine (Bitoxibacillin) a été décrite pour $D$ reticulatum (Terytze et Hofmann, 1986). Osman et al (1992) ont montré également un effet toxique de plusieurs formulations (Thuricide, SAN 401, SAN 402, SAN 415) sur l'escargot aquatique
Biomphalaria alexandrina (Ehrenberg). C'est pourquoi, il nous a semblé intéressant d'éprouver la toxicité de certains de ces produits, ainsi que l'action de diverses souches non formulées, produisant des $\delta$-entotoxines, sur les trois espèces de limaces d'importance économique, décrites précédemment.

\section{MATÉRIEL ET MÉTHODES}

\section{Origine et caractéristiques des produits}

Les produits testés sont soit des formulations commerciales, soit des souches sauvages non formulées.

Les souches non formulées proviennent de l'unité de recherche et de criblage de souches de $B$ thuringiensis de la station Inra de lutte biologique de La Minière. Elles sont cultivées dans $100 \mathrm{~mL}$ de milieu hydrolysat de caséine-tryptone à $30^{\circ} \mathrm{C}$, en agitation, pendant 48 heures (Lecadet et al, 1980). La présence des cristaux dans les cultures est vérifiée avant récolte par examen microscopique. La récolte du complexe spores-cristaux nécessite des centrifugations successives de 10 minutes, à $5000 \mathrm{rpm}$. La culture est concentrée 4 fois ( $25 \mathrm{~mL}$ final) ; le mélange spores-cristaux est conservé à $-20^{\circ} \mathrm{C}$ dans l'eau glycérolée à $1,7 \%$. L'analyse des protéines totales des souches est faite par électrophorèse en gel de polyacrylamide à 10,5\% (SDS-Page). Le dosage des protéines totales est réalisé selon la méthode de Bradford (1976). L'origine et les caractéristiques de ces différentes souches sont indiquées dans le tableau I.

La majorité des souches présentent des bandes de protéine "atypique», c'est-à-dire de poids moléculaire ne correspondant pas à ceux des protéines des souches communes de $B$ thuringiensis toxiques pour larves de lépidoptères, de coléoptères ou de diptères. Cependant, ces souches possèdent une activité insecticide sur la teigne des crucifères Plutella xyllostella $L$ (Chaufaux et al, 1991), un lépidoptère devenu résistant à plusieurs souches formulées de $B$ thuringiensis. Les souches dont les cristaux contiennent des protéines de poids moléculaire 40-45 kDa ont une très faible activité ou aucune activité sur de nombreuses espèces d'insectes testées appartenant à huit ordres (Chaufaux et al, 1991). La souche LM 303 est isolée de mollusques et synthétise des cristaux possédant des bandes de protéine à $130 \mathrm{kDa}$. II s'agit là d'un profil électrophorétique typique des souches actives sur larves de lépidoptères. Cependant, étant donné son origine, il nous a paru intéressant de la tester sur des limaces. Les cristaux de la souche LM 1599 II possèdent les bandes de protéine mixtes à 70-73 et $130 \mathrm{kDa}$. Ce profil est caractéristique des souches ayant une activité larvicide de coléoptères (bandes à 70-73 kDa) et de lépidoptères (bande à $130 \mathrm{kDa})$.

Les formulations commerciales : Bactospéine $6000 \AA$, Bactospéine $16000 \circledast$, Thuricide $5 \circledast$, provien- 
nent du laboratoire des bactéries entomopathogènes de l'Institut Pasteur. Biobit ${ }^{\circledR}$ et Dipel $($ sont des produits du commerce, homologués en France pour lutter contre certaines espèces de lépidoptères. Toutes ces spécialités sont formulées à partir du sérotype $3 a, 3 b$, de $B$ thuringiensis, variété kurstaki, dont le profil électrophorétique des cristaux est $70-130-132 \mathrm{kDa}$, à l'exception de Bactospéine $6000($ ), de sérotype 1, variété thuringiensis, dont le profil électrophorétique des cristaux est $132 \mathrm{kDa}$.

Enfin, deux produits mis en expérimentation contiennent de la $\beta$-exotoxine : Bitoxibacillin, de sérotype 1 , variété thuringiensis, est une préparation contenant deux toxines : une $\delta$-endotoxine et une $\beta$ exotoxine et provient de l'ex-Union soviétique. Le produit, appelé "surnageant $\mathrm{H} 1$ " dans nos essais, ne contient que la $\beta$-exotoxine (entre 0,25 et $0,30 \mathrm{~g} / \mathrm{L}$ ), car il s'agit du surnageant d'une culture bactérienne synthétisant cette toxine lors de la sporulation et provenant de l'Institut Pasteur (De Barjac et Lecadet, 1976).

Le produit appelé Israelensis $\mathrm{H} 14$ est la souche $\mathrm{H}$ 14 , variété israelensis. Cette souche produit un corps d'inclusion parasporal contenant des protéines d'une taille de 135, 128, 78, 72 et $27 \mathrm{kDa}$. Elle produit des toxines actives contre les larves de certains diptères. Elle a été cultivée dans les mêmes conditions que les souches provenant du laboratoire de La Minière.

\section{Matériel biologique}

Les limaces utilisées lors des essais appartiennent à trois espèces. Les individus des espèces $D$ reticulatum et $A$ distinctus proviennent d'une parcelle de luzerne de Colmar, tandis que ceux de l'espèce $L$ valentianus sont issus d'élevages en serre. Les limaces sont toutes de petite taille (jeunes) pour l'expérience 1 ou de taille moyenne pour les expériences 2 à 5 . Elles sont accoutumées aux conditions expérimentales pendant les 10 jours précédant les pulvérisations. Elles sont soumises au jeûne, 24 heures avant le début des expériences.

\section{Protocoles expérimentaux}

Les feuilles de laitue qui servent à l'alimentation des limaces sont traitées à la tour de pulvérisation (Burgerjon, 1956) à la dose de $1,7 \mathrm{mg}$ de suspension par centimètre carré (l'équivalent de $600 \mathrm{~L} / \mathrm{ha}$ ). Les doses des différentes préparations pulvérisées sont indiquées dans les tableaux des résultats (II, III, IV, V). Elles sont exprimées selon les produits (formulations commerciales, souches non formulées) en gramme de produit par litre ou en gramme de protéine par litre. Une quantité de feuilles, de surface équivalant à 25

Tableau I. Caractéristiques des souches non formulées de $B$ thuringiensis, provenant de l'Inra de La Minière.

Souches Provenance Espèces sensibles

LM 127 Déjection de lépidoptère

LM 303

LM 381

LM 409

LM 605B

LM $651 \mathrm{~A}$

LM 1060

LM 1212

LM 1271

LM 1273

LM 1274

LM 1497A

LM 1504A

LM 1506A

LM 1507A

LM 1599 II

LM 1602

LM 1613A

LM 1679

LM 1882

LM 2051

LM 2378

H 14

Mollusque

Déjection d'oiseau

Terre

Coléoptère

Orthoptère

Coléoptère

Coléoptère

Terre

Terre

Végétal

Déjection d'hyménoptère

Végétal

Végétal

Mollusque

Terre

Déjection de mammifère

Orthoptère

Terre

Terre

Terre

Terre

$\begin{array}{ll}- & 40-45 \\ \text { Lépidoptères } & 130 \\ - & 40-45 \\ - & 40-45 \\ \text { Plutella xyllostella } & 100-128 \\ - & 40-45 \\ - & 40-45 \\ \text { Plutella xyllostella } & 35 \\ \text { Plutella xyllostella } & 45-60 \\ \text { Plutella xyllostella } & 45-60 \\ \text { Plutella xyllostella } & 45-60 \\ - & 40-45 \\ - & 40-45 \\ \text { - } & 40-45 \\ \text { - } & 40-45 \\ \text { Coléoptères, lépidoptères } & 70-73 \text { et } 130 \\ \text { - } & 40-45 \\ \text { Plutella xyllostella } & 95-128 \\ \text { Plutella xyllostella } & 31-35-60-70-90 \\ \text { Plutella xyllostella } & 32-45-95-105 \\ \text { Plutella xyllostella } & 93-100-200 \\ \text { Plutella xyllostella } & 34-37-75 \\ \text { Diptères } & 27-72-78 \\ & 128-135\end{array}$

Taille des protéines du corps d'inclusion en $K D a$

128-135 
$\mathrm{cm}^{2}$, est ensuite placée dans une boîte de Pétri de 9 $\mathrm{cm}$ de diamètre, dont le fond est couvert d'un papier filtre humidifié. En fonction de l'expérience menée, des lots de 3 ou 5 limaces sont introduits dans chaque boîte. Les boîtes sont ensuite placées dans une enceinte climatique à $15 \pm 0,5^{\circ} \mathrm{C}$, avec une photophase de 14 heures (expériences 1 et 2), ou à $19 \pm 0,5$ ${ }^{\circ} \mathrm{C}$, avec une photophase de 12 heures (expériences 3 et 4), ou à $24^{\circ} \mathrm{C}$ et $22^{\circ} \mathrm{C} \pm 0,5^{\circ} \mathrm{C}$ pendant respectivement 12 heures de photophase et 12 heures de scotophase (expérience 5). Après 3 jours de consommation des feuilles de laitue traitées, les limaces sont alimentées avec des feuilles intactes. Chaque essai comporte un témoin traité à l'eau distillée. Dans les boîtes contenant des granulés molluscicides de référence, les granulés sont également remplacés, après trois jours, par des feuilles de laitue intactes.

La consommation de laitue et la mortalité des limaces sont vérifiées quotidiennement pendant les 9 jours qui suivent le traitement.

\section{Expérience 1}

Bactospéine 6000, Bactospéine 16000, Dipel, Thuricide 5 sont pulvérisés à des doses proches de celles recommandées pour lutter contre les larves de lépidoptères. L'efficacité du Bitoxibacillin 5 est jugée dans les mêmes conditions. Chaque traitement est réalisé avec cinq lots de cinq jeunes $D$ reticulatum.

\section{Expérience 2}

Le surnageant $\mathrm{H} 1$ est expérimenté sur dix lots de cinq $D$ reticulatum.

\section{Expérience 3}

Les suspensions de spores et de cristaux de cinq des souches non formulées, contenant $1 \mathrm{~g}$ de protéine/L, sont étudiées en comparaison avec des solutions de molluscicide (Mesurol ${ }^{\circledR} 50$, contenant $50 \%$ de méthiocarbe) aux doses de $0,2,0,6$ et $1 \mathrm{~g} / \mathrm{L}$. Chaque traitement est réalisé avec six lots de cinq $A$ distinctus.

\section{Expérience 4}

L'action de suspensions de cristaux non formulées, contenant $2 \mathrm{~g}$ de protéine/L, est comparée à celle de deux granulés molluscicides de référence contenant respectivement $4 \%$ de méthiocarbe (Mesuro $\left.\right|^{\circledR}$ ) et $5 \%$ de bensultap (Malice ${ }^{\circledR}$ ). Les cristaux des 18 souches testées ont été dissous dans un tampon de bicarbonate de sodium à $\mathrm{pH} 10$. Le $\mathrm{pH}$ des suspensions a ensuite été ramené à 7 , pour éviter de provoquer la mort des limaces du fait du pH élevé. Le produit commercial Biobit a été examiné à 2 doses, 0,4 et $4 \mathrm{~g} / \mathrm{L}$, qui encadrent celle préconisée à l'emploi $(1,6 \mathrm{~g} / \mathrm{L})$. Les produits molluscicides de référence sont placés dans les boîtes de Pétri à raison de trois granulés par boîte. Dix lots de $3 L$ valentianus sont utilisés par traitement. Dans le cas du molluscicide à $4 \%$ de méthiocarbe, deux répétitions ont été effectuées (Mesurol 1 et 2).

\section{Expérience 5}

La toxicité de préparations commerciales de $B$ thuringiensis, dont certaines se sont montrées toxiques envers $D$ reticulatum (Terytze et Hofmann, 1986), est comparée à celle d'un granulé molluscicide de référence contenant $4 \%$ de méthiocarbe (Mesurol). L'action de solutions de Bitoxibacillin, Dipel, Bactospéine 16000 , Biobit, préparées à la dose de $1 \%(10 \mathrm{~g} / \mathrm{L})$, est étudiée selon le même protocole que celui de l'expérience 4 . Dix lots de trois $D$ reticulatum et de trois $L$ valentianus sont utilisés par traitement.

\section{RÉSULTATS ET DISCUSSION}

\section{Sensibilité de $\mathrm{D}$ reticulatum aux produits commerciaux formulés à partir de $\mathrm{B}$ thuringiensis, et aux produits contenant de la $\beta$-exotoxine}

Les individus de $D$ reticulatum ont entièrement dévoré les feuilles de laitue traitées, mais aucun effet toxique significatif n'a été décelé, dans les expériences 1,2 et 5 (tableaux II et V). Terytze et Hofmann (1986) constatent une importante mortalité d'individus de $D$ reticulatum après l'ingestion de feuilles de Gerbera pulvérisées avec des solutions contenant de la $\beta$-exotoxine (Bitoxibacillin) ou n'en contenant pas (Dendrobacillin, Thuricide-HP et Dipel). Cependant, l'absence de lot témoin ne permet pas de distinguer la mortalité naturelle des animaux de celle due aux souches de $B$ thuringiensis. Nous avons reproduit, avec des solutions pulvérisées sur des feuilles de laitue et des $D$ reticulatum de taille moyenne, une partie des tests de Terytze et Hofmann (dose des produits de $1 \%$, température de $22-24{ }^{\circ} \mathrm{C}$ ), sans observer de mortalité. Ces auteurs ayant utilisé des limaces adultes, il est possible qu'elles soient mortes naturellement, car chez $D$ reticulatum les individus meurent peu de temps après la période de reproduction. Les conditions de température élevées pour cette espèce $\left(22-24^{\circ} \mathrm{C}\right)$ pourraient être une autre cause de mortalité observée. Par ailleurs, une partie de l'expérimentation rapportée par ces auteurs a été faite par injection de $B$ thuringiensis et n'est pas comparable à celle-ci. 
Tableau II. Effet de la consommation de feuilles de laitue pulvérisées avec des suspensions de $B$ thuringiensis, chez $D$ reticulatum. Expérience 1 : cinq lots de cinq limaces, expérience 2 : dix lots de cinq limaces, par produit.

\begin{tabular}{|c|c|c|c|c|c|}
\hline Expérience & Produits & Sérotype & $\begin{array}{l}\text { Nombre } \\
\text { de limaces }\end{array}$ & $\begin{array}{l}\text { Dose } \\
(g / L)\end{array}$ & $\begin{array}{l}\text { Nombre } \\
\text { de morts à } 9 \text { jours }\end{array}$ \\
\hline \multirow[t]{6}{*}{1} & Témoin & - & 25 & & 5 \\
\hline & Bactospéine 6000 & 1 & 25 & 1,5 & 1 \\
\hline & Bactospéine & $3 a 3 b$ & 25 & 0,6 & 0 \\
\hline & Dipel & $3 a 3 b$ & 25 & 0,3 & 0 \\
\hline & Thuricide 5 & $3 a 3 b$ & 25 & 0,09 & 0 \\
\hline & Bitoxibacillin 5 & 1 & 25 & 1 & 2 \\
\hline \multirow[t]{2}{*}{2} & Témoin & & 50 & & 8 \\
\hline & Surnageant $\mathrm{H} 1$ & & 50 & $0,25-0,30$ & 8 \\
\hline
\end{tabular}

\section{Sensibilité de A distinctus aux souches non formulées de $\mathrm{B}$ thuringiensis}

Aucune des cinq souches, isolées d'échantillons de terre et d'insectes et présentant des profils électrophorétiques atypiques, n'a provoqué de mortalité significative par rapport au lot témoin (tableau III).

Seule l'ingestion des feuilles de laitues traitées avec du Mesurol 50 à $0,6 \mathrm{~g} / \mathrm{L}$ et à $1 \mathrm{~g} / \mathrm{L}$ a provoqué une mortalité nettement supérieure à celle des témoins et des autres traitements. La solution de Mesurol 50 à $0,6 \mathrm{~g} / \mathrm{L}$ s'est montrée la plus toxique et a provoqué la mortalité de toutes les limaces horticoles au bout de 5 jours.

\section{Sensibilité de $L$ valentianus aux souches non formulées et aux produits commerciaux formulés à partir de $\mathrm{B}$ thuringiensis}

Les souches de $B$ thuringiensis ont été isolées d'échantillons de nature très diverse : terre, insectes, déjections, végétaux, mollusques. La plupart présente un profil électrophorétique atypique. Seules les deux souches suivantes peuvent être considérées comme «normales». La souche LM 303, qui a une bande à $130 \mathrm{kDa}$, a une protéine active sur larves de lépidoptères, mais cette souche a été isolée à partir d'un mollusque terrestre. La souche LM 1599 II a la particularité de synthétiser des cristaux présentant deux activités larvicides : l'une sur lépidoptères (130 kDa) et l'autre sur coléoptères (70-73 kDa).

L'ensemble des $L$ valentianus ayant consommé des feuilles de laitue traitées est vivant 9 jours après le traitement, excepté quelques indi- vidus répartis dans différentes souches (tableaux IV et V). Un seul individu a survécu en présence des granulés de méthiocarbe dans les expériences 4 et 5 , alors qu'avec les granulés de bensultap 15 individus sur 30 sont restés vivants.

Les expériences que nous rapportons montrent que plusieurs souches de $B$ thuringiensis ayant des activités insecticides différentes, ou pas d'activité insecticide encore démontrée, n'ont pas d'action par ingestion sur les trois espèces de limaces examinées. Ces résultats confirment la spécificité d'action des souches bactériennes et sont en contradiction avec les effets toxiques observés par Terytze et Hofmann (1986) chez $D$ reticulatum. L'activité toxique de produits formulés à partir de $B$ thuringiensis sur l'escargot aquatique $B$ alexandrina et rapportée par Osman et al (1992) est due, à l'exception d'un produit

Tableau III. Effet de la consommation de feuilles de laitue pulvérisées avec des suspensions de $B$ thuringiensis chez $A$ distinctus (six lots de cinq limaces par produit), en comparaison avec des solutions molluscicides (Mesurol 50) à différentes doses (expérience 3).

$\begin{array}{cccc}\text { Produits } & \begin{array}{c}\text { Nombre } \\ \text { de limaces }\end{array} & \begin{array}{c}\text { Doses } \\ \text { utilisées }(g / L)\end{array} & \begin{array}{c}\text { Nombre de } \\ \text { morts à } 9 \text { jours }\end{array}\end{array}$

$\begin{array}{lccc}\text { Témoin 1 } & 30 & - & 0 \\ \text { Témoin 2 } & 30 & - & 0 \\ \text { Témoin 3 } & 30 & - & 0 \\ \text { Mesurol 50 } & 30 & 0,2 & 2 \\ \text { Mesurol 50 } & 30 & 0,6 & 30 \\ \text { Mesurol 50 } & 30 & 1 & 20 \\ \text { LM 409 } & 30 & 1 & 4 \\ \text { LM 651A } & 30 & 1 & 3 \\ \text { LM 1060 } & 30 & 1 & 1 \\ \text { LM 1212 } & 30 & 1 & 5 \\ \text { LM 1273 } & 30 & 1 & 0\end{array}$


Tableau IV. Effets comparés de la consommation de feuilles de laitue pulvérisées avec des suspensions de $B$ thuringiensis et de celle de deux types de granulés molluscicides (Mesurol et Malice), chez $L$ valentianus (dix lots de trois limaces par produit) (expérience 4).

\begin{tabular}{|c|c|c|c|}
\hline Produits & $\begin{array}{c}\text { Nombre de } \\
\text { limaces }\end{array}$ & $\begin{array}{l}\text { Doses } \\
\text { utilisées }\end{array}$ & $\begin{array}{c}\text { Nombre de } \\
\text { morts à } 9 \text { jours }\end{array}$ \\
\hline
\end{tabular}

$\begin{array}{llcc}\text { Témoin 1 } & 30 & - & 0 \\ \text { Témoin 2 } & 30 & - & 0 \\ \text { Mesurol 1 } & 30 & 3 \text { granulés } & 29 \\ \text { Mesurol 2 } & 30 & 3 \text { granulés } & 29 \\ \text { Malice } & 30 & 3 \text { granulés } & 15 \\ \text { LM 127 } & 30 & 2 \mathrm{~g} / \mathrm{L} & 0 \\ \text { LM 303 } & 30 & 2 \mathrm{~g} / \mathrm{L} & 0 \\ \text { LM 381 } & 30 & 2 \mathrm{~g} / \mathrm{L} & 0 \\ \text { LM 605B } & 30 & 2 \mathrm{~g} / \mathrm{L} & 0 \\ \text { LM 1271 } & 30 & 2 \mathrm{~g} / \mathrm{L} & 0 \\ \text { LM 1274 } & 30 & 2 \mathrm{~g} / \mathrm{L} & 1 \\ \text { LM 1497A } & 30 & 2 \mathrm{~g} / \mathrm{L} & 0 \\ \text { LM 1504A } & 30 & 2 \mathrm{~g} / \mathrm{L} & 0 \\ \text { LM 1506A } & 30 & 2 \mathrm{~g} / \mathrm{L} & 0 \\ \text { LM 1507A } & 30 & 2 \mathrm{~g} / \mathrm{L} & 0 \\ \text { LM 1599 II } & 30 & 2 \mathrm{~g} / \mathrm{L} & 0 \\ \text { LM 1602 } & 30 & 2 \mathrm{~g} / \mathrm{L} & 0 \\ \text { LM 1613A } & 30 & 2 \mathrm{~g} / \mathrm{L} & 2 \\ \text { LM 1679 } & 30 & 2 \mathrm{~g} / \mathrm{L} & 1 \\ \text { LM 1882 } & 30 & 2 \mathrm{~g} / \mathrm{L} & 0 \\ \text { LM 2051 } & 30 & 2 \mathrm{~g} / \mathrm{L} & 0 \\ \text { LM 2378 } & 30 & 2 \mathrm{~g} / \mathrm{L} & 0 \\ \text { Israelensis, H14 } & 30 & 2 \mathrm{~g} / \mathrm{L} & 0 \\ \text { Biobit } & 30 & 0,4 \mathrm{~g} / \mathrm{L} & 0 \\ \text { Biobit } & 30 & 4 \mathrm{~g} / \mathrm{L} & 0 \\ & & & \end{array}$

Tableau V. Effets comparés de la consommation de feuilles de laitue pulvérisées avec des suspensions de $B$ thuringiensis (dose de $10 \mathrm{~g} / \mathrm{L}$ ) et de celle de granulés molluscicides (Mesurol), chez $D$ reticulatum et $L$ valentianus (dix lots de trois limaces par produit) (expérience 5).

$\begin{array}{cc}\text { Produits } & \begin{array}{c}\text { Nombre de } \\ \text { limaces }\end{array} \\ & \text { Nombre de morts } \\ \text { à } 9 \text { jours }\end{array}$

$D$ reticulatum $L$ valentianus

$\begin{array}{lccc} & & & 0 \\ \text { Témoin } & 30 & 1 & 29 \\ \text { Mesurol } & 30 & 29 & 0 \\ \text { Bitoxibacillin 5 } & 30 & 0 & 0 \\ \text { Dipel } & 30 & 0 & 0 \\ \text { Bactospéine 16000 } & 30 & 0 & 1 \\ \text { Biobit } & 30 & 0 & \end{array}$

commercial (Thuricide), à l'activité de préparations expérimentales pour lesquelles il n'y a pas d'indication de composition. II serait donc intéressant d'étudier sur les limaces l'action de ces dernières, si celles-ci ne contiennent pas de $\beta$-exotoxine.

\section{REMERCIEMENTS}

Nous tenons à remercier Mlle de Barjac (Institut Pasteur, Paris) pour nous avoir fourni plusieurs souches et pour ses conseils et M Bauer (société Novo Nordisk) pour la fourniture du produit Biobit.

\section{RÉFÉRENCES}

Bradford MM (1976) A rapid and sensitive method for the quantification of microgram quantities of protein utilizing the principle of protein-dye binding. Ann Biochem 72, 248-254

Burgerjon A (1956) Pulvérisation et poudrage au laboratoire par des préparations pathogènes insecticides. Ann Epiphyt 4, 675-684

Burgerjon A (1972) Quelques effets physiologiques de la toxine thermostable de Bacillus thuringiensis sur le doryphore Leptinotarsa decemlineata. Ent Exp App/ 15, 112-127

Chaufaux J, Marchal M, Marchal D, Gilois N (1991) Recherche de nouvelles souches de Bacillus thuringiensis Berliner : activité de la souche LM 63 sur des coléoptères Chrysomelidae. Rencontres caraïbes en lutte biologique, Guadeloupe 5-7 nov 1990. Les colloques de l'Inra 58, 315-324

De Barjac H, Lecadet MM (1976) Dosage biochimique de l'exotoxine thermostable de $B$ thuringiensis d'après l'inhibition d'ARN-polymérases bactériennes. C R Séances Acad Sci (Paris) Sér D 282, 2119-2122

Hommay G, Lavanceau P (1986) Les différentes espèces de limaces présentes en grandes cultures. Phytoma - Déf des Cult, février 1986, 19-22

Lecadet MM, Blondel MO, Ribier J (1980) Generalized transduction in Bacillus thuringiensis var Berliner 1715, using bacteriophage CP 54 Ber. J Gen Microbiol 121, 203-212

Lereclus D, Sanchis V (1989) Les $\delta$-endotoxines de Bacillus thuringiensis à propriétés insecticides. $C R$ Acad Agri Fr 75, 117-126

Moens R (1980) Le problème des limaces dans la protection des végétaux. Rev Agric Belg 33, 117-132

Osman GY, Mohamed AM, Jamel-al-Layl K (1992) Über die molluscizide Wirkung von Bacillus thuringiensis-Präparaten zur biologischen Bekämpfung von Biomphalaria alexandrina-Schnecken, den Zwischenwirten des Bilharziose-Saugwurms Schistosoma mansoni in Saudi Arabia. Anz Schädlingskde Pflanzenschutz Umweltschutz 65, $67-70$ 
Poinar GO, Thomas GM, Lighthart B (1990) Bioassay to determine the effect of commercial preparations of Bacillus thuringiensis on entomogenous rhabditoid nematodes. Agric Ecosyst Environ 30, 195202

Rajnchapel-Messaï J (1993) Bacillus thuringiensis, les insectes font de la résistance. Biofutur, octobre 1993, 33-38

Royalty RN, Hall FR, Taylor RA (1990) Effects of Thuringiensin on Tetranychus urticae (Acari: Tetranychidae) mortality, fecondity, and feeding. J Econ Entomol 83, 792-798
South A (1992) Terrestrial Slugs. Biology, Ecology and Control. Chapman \& Hall, Londres, $428 \mathrm{p}$

Terytze K, Hofmann G (1986) Die Wirkung von Bakterienpräparaten (Bacillus thuringiensis Berliner) zur Bekämpfung von Nacktschnecken in Gerbera Beständen. Arch Phytopathol Pflanzenschutz 41, 361-363

Waldén HW (1960) On two anthropochorous terrestrial gastropods, Limax valentianus Férussac and Deroceras caruanae (Pollonera), found in Sweden. Göteborgs Kungl Vetensk Vitterhets Samhalles Handl F 6, Ser B, 8, 42-47 